$\mathrm{J}$ o u r n a l of

Mathematics

and Applications

JMA No 40, pp 149-159 (2017)

\title{
Ergodic Properties of Random Infinite Products of Nonexpansive Mappings
}

\author{
Simeon Reich and Alexander J. Zaslavski
}

\begin{abstract}
In this paper we are concerned with the asymptotic behavior of random (unrestricted) infinite products of nonexpansive selfmappings of closed and convex subsets of a complete hyperbolic space. In contrast with our previous work in this direction, we no longer assume that these subsets are bounded. We first establish two theorems regarding the stability of the random weak ergodic property and then prove a related generic result. These results also extend our recent investigations regarding nonrandom infinite products.
\end{abstract}

AMS Subject Classification: 47H09, 47H14, 54E35.

Keywords and Phrases: Complete metric space; Hyperbolic space; Infinite product; Nonexpansive mapping; Random weak ergodic property.

\section{Introduction and preliminaries}

It is well known that (unrestricted) infinite products of operators find applications in many areas of mathematics (see, for example, $[1,2,3,4,5,9,11]$ and the references mentioned therein). In this paper we establish weak ergodic theorems concerning the asymptotic behavior of random (that is, unrestricted) infinite products of nonexpansive mappings on closed and convex subsets of a Banach space which are not necessarily bounded. These theorems continue our previous work [9], where we assumed that the mappings under consideration act on a bounded set. They also extend the results of [12], which were obtained for nonrandom infinite products.

More precisely, our paper contains three theorems. The first two show that if the random weak ergodic property (see the definition below) holds for a sequence of nonexpansive mappings, then it is stable under small perturbations of these mappings. In our second theorem the perturbed mappings are also nonexpansive, while in the first one the perturbations can be arbitrary. The third theorem establishes the random weak ergodic property for a generic sequence of nonexpansive mappings. Namely, we show that in an appropriate space of sequences of nonexpansive mappings there exists

COPYRIGHT (C) by Publishing House of Rzeszów University of Technology P.O. Box 85, 35-959 Rzeszów, Poland 
a subset which is a countable intersection of open and everywhere dense sets such that each sequence belonging to this subset has the random weak ergodic property. Such an approach is common in nonlinear analysis $[9,10,11]$. Thus, instead of considering a certain convergence property for a single sequence of operators, we investigate it for a space of all such sequences equipped with some natural metric, and show that this property holds for most of these sequences in the sense of Baire category. This allows us to establish convergence without restrictive assumptions on the space and on the operators themselves.

As a matter of fact, it turns out that our results also hold for nonexpansive selfmappings of closed and convex sets in complete hyperbolic spaces, an important class of metric spaces the definition of which we now recall for the reader's convenience.

Let $(X, \rho)$ be a metric space and let $R^{1}$ denote the real line. We say that a mapping $c: R^{1} \rightarrow X$ is a metric embedding of $R^{1}$ into $X$ if $\rho(c(s), c(t))=|s-t|$ for all real $s$ and $t$. The image of $R^{1}$ under a metric embedding is called a metric line. The image of a real interval $[a, b]=\left\{t \in R^{1}: a \leq t \leq b\right\}$ under such a mapping is called a metric segment.

Assume that $(X, \rho)$ contains a family $M$ of metric lines such that for each pair of distinct points $x$ and $y$ in $X$, there is a unique metric line in $M$ which passes through $x$ and $y$. This metric line determines a unique metric segment joining $x$ and $y$. We denote this segment by $[x, y]$. For each $0 \leq t \leq 1$, there is a unique point $z$ in $[x, y]$ such that

$$
\rho(x, z)=t \rho(x, y) \text { and } \rho(z, y)=(1-t) \rho(x, y) .
$$

This point will be denoted by $(1-t) x \oplus t y$. We say that $X$, or more precisely $(X, \rho, M)$, is a hyperbolic space if

$$
\rho\left(\frac{1}{2} x \oplus \frac{1}{2} y, \frac{1}{2} x \oplus \frac{1}{2} z\right) \leq \frac{1}{2} \rho(y, z)
$$

for all $x, y$ and $z$ in $X$. An equivalent requirement is that

$$
\rho\left(\frac{1}{2} x \oplus \frac{1}{2} y, \frac{1}{2} w \oplus \frac{1}{2} z\right) \leq \frac{1}{2}(\rho(x, w)+\rho(y, z))
$$

for all $x, y, z$ and $w$ in $X$. This inequality, in its turn, implies that

$$
\rho((1-t) x \oplus t y,(1-t) w \oplus t z) \leq(1-t) \rho(x, w)+t \rho(y, z)
$$

for all points $x, y, z$ and $w$ in $X$, and all numbers $0 \leq t \leq 1$.

It is clear that all normed linear spaces are hyperbolic in this sense. A discussion of more examples of hyperbolic spaces and, in particular, of the Hilbert ball with the hyperbolic metric can be found, for example, in $[6,7,8]$.

We call a set $K \subset X \rho$-convex if $[x, y] \subset K$ for all $x$ and $y$ in $K$.

Suppose that $(X, \rho, M)$ is a complete hyperbolic space and that $K$ is a nonempty, closed and $\rho$-convex subset of the space $X$. Denote by $\mathcal{A}$ the collection of all operators $T: K \rightarrow K$ which satisfy

$$
\rho(T(x), T(y)) \leq \rho(x, y) \text { for all } x, y \in K .
$$


Denote by $\mathcal{M}$ the set of all sequences of operators $\left\{T_{i}\right\}_{i=1}^{\infty} \subset \mathcal{A}$. For every sequence of operators $\left\{B_{i}\right\}_{i=1}^{\infty} \in \mathcal{M}$ and every pair of integers $p>n \geq 1$, we define compositions of the corresponding operators by

$$
\prod_{i=n}^{p} B_{i}:=B_{p} \cdots B_{n} .
$$

For every point $x \in K$ and every positive number $r$, set

$$
B(x, r):=\{y \in K: \rho(x, y) \leq r\} .
$$

Fix a point $\theta \in K$. We equip the set $\mathcal{M}$ with the uniformity determined by the following base:

$$
\begin{gathered}
\mathcal{U}(n):=\left\{\left(\left\{A_{t}\right\}_{t=1}^{\infty},\left\{B_{t}\right\}_{t=1}^{\infty}\right) \in \mathcal{M} \times \mathcal{M}:\right. \\
\left.\rho\left(A_{t}(x), B_{t}(x)\right) \leq n^{-1} \text { for all } x \in B(\theta, n) \text { and all integers } t \geq 1\right\},
\end{gathered}
$$

where $n \geq 1$ is an integer. It is not difficult to see that the uniform space $\mathcal{M}$ is metrizable (by a metric $d$ ) and complete. In principle, one can obtain an explicit expression for this metric $d$, but we do not need it because in our case it is more convenient to use the uniformity itself.

Denote by $I: K \rightarrow K$ the identity operator; that is, $I(x)=x$ for all $x \in K$.

In this paper we are interested in those sequences of mappings in $\mathcal{M}$ which are uniformly bounded on bounded sets.

Proposition 1.1. Let $\left\{A_{t}\right\}_{t=1}^{\infty} \in \mathcal{M}, x \in K$ and assume that $\left\{A_{t}(x)\right\}_{t=1}^{\infty}$ is a bounded sequence. Then for every $y \in K$, the sequence $\left\{A_{t}(y)\right\}_{t=1}^{\infty}$ is bounded and

$$
\sup \left\{\rho\left(\theta, A_{t}(y)\right): t=1,2, \ldots\right\} \leq \sup \left\{\rho\left(\theta, A_{t}(x)\right): t=1,2, \ldots\right\}+\rho(x, y) .
$$

Proof. Clearly, the real sequence $\left\{\rho\left(\theta, A_{t}(x)\right)\right\}_{t=1}^{\infty}$ is bounded. Let $y \in K$. Then in view of (1.1), for every integer $t \geq 1$,

$$
\rho\left(\theta, A_{t}(y)\right) \leq \rho\left(\theta, A_{t}(x)\right)+\rho\left(A_{t}(x), A_{t}(y)\right) \leq \rho\left(\theta, A_{t}(x)\right)+\rho(x, y) .
$$

Proposition 1.1 now follows immediately.

We denote by $\mathcal{M}_{n}$ the set of all sequences $\left\{A_{t}\right\}_{t=1}^{\infty} \in \mathcal{M}$ such that the sequence $\left\{A_{t}(\theta): t=1,2, \ldots\right\}$ is bounded. Clearly, $\mathcal{M}_{n}$ is a closed and open subset of the complete metric space $(\mathcal{M}, d)$. In this paper we focus on the complete metric space $\left(\mathcal{M}_{n}, d\right)$.

For every point $z \in K$ and every nonempty set $D \subset K$, set

$$
\rho(z, D):=\inf \{\rho(z, \xi): \xi \in D\} .
$$

We say that a sequence of mappings $\left\{A_{t}\right\}_{t=1}^{\infty} \in \mathcal{M}$ possesses the weak ergodic property [12] (WEP, for short) if for every pair of positive numbers $\epsilon, s$, there exists an integer $n_{0} \geq 1$ such that for every pair of points $x, y \in B(\theta, s)$, we have

$$
\rho\left(A_{n_{0}} \cdots A_{1}(x), A_{n_{0}} \cdots A_{1}(y)\right) \leq \epsilon .
$$


In [12] we consider the space $\mathcal{M}$ equipped with the uniformity determined by the base

$$
\begin{gathered}
\tilde{\mathcal{U}}(n):=\left\{\left(\left\{A_{t}\right\}_{t=1}^{\infty},\left\{B_{t}\right\}_{t=1}^{\infty}\right) \in \mathcal{M} \times \mathcal{M}:\right. \\
\left.\rho\left(A_{t}(x), B_{t}(x)\right) \leq n^{-1} \text { for all } x \in B(\theta, n) \text { and all } t=1, \ldots, n\right\},
\end{gathered}
$$

where $n$ is a natural number, and show that most sequences in $\mathcal{M}$, in the sense of Baire category, have the WEP and that the WEP is stable under small perturbations.

We say that a sequence of mappings $\left\{A_{t}\right\}_{t=1}^{\infty} \in \mathcal{M}$ possesses the random weak ergodic property (RWEP, for short) if for every pair of positive numbers $\epsilon, s$, there exists an integer $n_{0} \geq 1$ such that for every pair of points $x, y \in B(\theta, s)$ and every mapping $r:\left\{1, \ldots, n_{0}\right\} \rightarrow\{1,2, \ldots\}$, we have

$$
\rho\left(A_{r\left(n_{0}\right)} \cdots A_{r(1)}(x), A_{r\left(n_{0}\right)} \cdots A_{r(1)}(y)\right) \leq \epsilon .
$$

In [13] we continue to study the space $\mathcal{M}$ with the uniformity introduced in [12] and show that most sequences in $\mathcal{M}$, in the sense of Baire category, do not have the RWEP and that they display, in fact, chaotic asymptotic behavior. In the present paper we show, on the other hand, that the RWEP does hold generically in the complete metric space $\left(\mathcal{M}_{n}, d\right)$. We begin with the following stability result.

\section{First stability result}

Theorem 2.1. Assume that a sequence of mappings $\left\{A_{t}\right\}_{t=1}^{\infty} \in \mathcal{M}$ possesses the $R W E P$ and let $\epsilon, s$ be positive numbers. Then there exists an integer $n_{0} \geq 1$ such that for every natural number $n \geq n_{0}$, there exists a number $\delta>0$ such that for every mapping $r:\{1,2, \ldots, n\} \rightarrow\{1,2, \ldots\}$ and every pair of sequences $\left\{x_{i}\right\}_{i=0}^{n},\left\{y_{i}\right\}_{i=0}^{n} \subset K$ satisfying

$$
x_{0}, y_{0} \in B(\theta, s)
$$

and

$$
\rho\left(x_{i+1}, A_{r(i+1)}\left(x_{i}\right)\right) \leq \delta, \rho\left(y_{i+1}, A_{r(i+1)}\left(y_{i}\right)\right) \leq \delta
$$

for all integers $i=0, \ldots, n-1$, the inequality $\rho\left(x_{i}, y_{i}\right) \leq \epsilon$ is valid for all integers $i=n_{0}, \ldots, n$.

Proof. By definition, there exists an integer $n_{0} \geq 1$ such that the following property holds:

(i) for every mappings $r:\left\{1, \ldots, n_{0}\right\} \rightarrow\{1,2, \ldots\}$ and every pair of points

$$
x, y \in B(\theta, s),
$$

we have

$$
\rho\left(A_{r\left(n_{0}\right)} \cdots A_{r(1)}(x), A_{r\left(n_{0}\right)} \cdots A_{r(1)}(y)\right) \leq \epsilon / 2 .
$$

Let $n \geq n_{0}$ be a natural number and let

$$
\delta=\epsilon(4 n)^{-1} .
$$


Assume that

$$
r:\{1, \ldots, n\} \rightarrow\{1,2, \ldots\}
$$

and that two sequences

$$
\left\{x_{i}\right\}_{i=0}^{n},\left\{y_{i}\right\}_{t=0}^{n} \subset K
$$

satisfy inclusion (2.1) and inequalities (2.2). It then follows from property (i) and (2.1) that

$$
\rho\left(A_{r\left(n_{0}\right)} \cdots A_{r(1)}\left(x_{0}\right), A_{r\left(n_{0}\right)} \cdots A_{r(1)}\left(y_{0}\right)\right) \leq \epsilon / 2 .
$$

When combined with (1.1), this inequality implies that

$$
\rho\left(A_{r(p)} \cdots A_{r(1)}\left(x_{0}\right), A_{r(p)} \cdots A_{r(1)}\left(y_{0}\right)\right) \leq \epsilon / 2 \text { for all } p=n_{0}, \ldots, n .
$$

Let

$$
\begin{gathered}
\left\{z_{i}\right\}_{i=0}^{n} \in\left\{\left\{x_{i}\right\}_{i=0}^{n},\left\{y_{i}\right\}_{i=0}^{n}\right\}, \\
\xi_{0}=z_{0}
\end{gathered}
$$

and let for all $i=0, \ldots, n-1$,

$$
\xi_{i+1}=A_{r(i+1)}\left(\xi_{i}\right) .
$$

We claim that for all integers $i=0, \ldots, n$, we have

$$
\rho\left(z_{i}, \xi_{i}\right) \leq i \delta .
$$

We first note that it follows from (2.6) that inequality (2.8) is valid for $i=0$.

Assume now that $i<n$ is a nonnegative integer and that inequality (2.8) is true. By (1.1), (2.2), (2.5), (2.7) and (2.8), we have

$$
\begin{gathered}
\rho\left(z_{i+1}, \xi_{i+1}\right)=\rho\left(z_{i+1}, A_{r(i+1)}\left(\xi_{i}\right)\right) \\
\leq \rho\left(z_{i+1}, A_{r(i+1)}\left(z_{i}\right)\right)+\rho\left(A_{r(i+1)}\left(z_{i}\right), A_{r(i+1)}\left(\xi_{i}\right)\right) \\
\leq \delta+\rho\left(z_{i}, \xi_{i}\right) \leq(i+1) \delta .
\end{gathered}
$$

Thus we have shown by induction that inequality (2.8) is indeed true for all integers $i=0, \ldots, n$, as claimed. When combined with $(2.5)-(2.7)$, this implies that for all integers $i=1, \ldots, n$, we have

$$
\begin{array}{r}
\rho\left(x_{i}, A_{r(i)} \cdots A_{r(1)}\left(x_{0}\right)\right) \leq i \delta \\
\rho\left(y_{i}, A_{r(i)} \cdots A_{r(1)}\left(y_{0}\right)\right) \leq i \delta
\end{array}
$$

and

$$
\rho\left(x_{i}, y_{i}\right) \leq \rho\left(A_{r(i)} \cdots A_{r(1)}\left(x_{0}\right), A_{r(i)} \cdots A_{r(1)}\left(y_{0}\right)\right)+2 i \delta
$$

It now follows from $(1.1),(2.3),(2.4)$ and (2.9) that for all integers $i=n_{0}, \ldots, n$,

$$
\rho\left(x_{i}, y_{i}\right) \leq \rho\left(A_{r(i)} \cdots A_{r(1)}\left(x_{0}\right), A_{r(i)} \cdots A_{r(1)}\left(y_{0}\right)\right)+2 n \delta \leq \epsilon / 2+\epsilon / 2 .
$$

This completes the proof of Theorem 2.1. 


\section{Second stability result}

Theorem 3.1. Assume that a sequence of mappings $\left\{A_{t}\right\}_{t=1}^{\infty} \in \mathcal{M}_{n}$ possesses the $R W E P$ and let $\epsilon, s$ be positive numbers. Then there exists an integer $n_{0} \geq 1$ and a neighborhood $\mathcal{U}$ of $\left\{A_{t}\right\}_{t=1}^{\infty}$ in $\mathcal{M}$ such that for every sequence of mappings $\left\{B_{t}\right\}_{t=1}^{\infty} \in \mathcal{U}$, every mapping $r:\{1,2, \ldots\} \rightarrow\{1,2, \ldots\}$, every pair of points $x, y \in B(\theta, s)$ and every natural number $n \geq n_{0}$, the inequality

$$
\rho\left(B_{r(n)} \cdots B_{r(1)}(x), B_{r(n)} \cdots B_{r(1)}(y)\right) \leq \epsilon
$$

is true.

Proof. Theorem 2.1 implies that there exist an integer $n_{0} \geq 1$ and a real number $\delta \in(0,1)$ such that the following property holds:

(i) for every mapping $r:\left\{1, \ldots, n_{0}\right\} \rightarrow\{1,2, \ldots\}$ and every pair of sequences $\left\{x_{i}\right\}_{t=0}^{n_{0}},\left\{y_{i}\right\}_{t=0}^{n_{0}} \subset K$ satisfying

$$
x_{0}, y_{0} \in B(\theta, s)
$$

and

$$
\rho\left(x_{i+1}, A_{r(i+1)}\left(x_{i}\right)\right) \leq \delta, \rho\left(y_{i+1}, A_{r(i+1)}\left(y_{i}\right)\right) \leq \delta
$$

for all $i=0, \ldots, n_{0}-1$, we have $\rho\left(x_{n_{0}}, y_{n_{0}}\right) \leq \epsilon$.

Define

$$
F_{0}:=B(\theta, s)
$$

and for all $i=1, \ldots, n_{0}-1$, define

$$
F_{i+1}:=\left\{y \in K: \rho\left(y, \cup\left\{A_{p}\left(F_{i}\right): p=1,2, \ldots\right\}\right) \leq 1\right\} .
$$

We now show by induction that all the sets $F_{0}, \ldots, F_{n_{0}}$ are bounded. In view of (3.1), $F_{0}$ is clearly bounded. Assume that $i<n_{0}$ is a nonnegative integer and that the set $F_{i}$ is bounded. Then there exists $M_{0}>0$ such that

$$
\rho(\theta, z) \leq M_{0} \text { for all } z \in F_{i} .
$$

By (3.3) and Proposition 1.1, for each $y \in F_{i}$,

$$
\begin{gathered}
\sup \left\{\rho\left(\theta, A_{p}(y)\right): p=1,2, \ldots\right\} \\
\leq \sup \left\{\rho\left(\theta, A_{p}(\theta)\right): p=1,2, \ldots\right\}+\rho(\theta, y) \\
\leq \sup \left\{\rho\left(\theta, A_{p}(\theta)\right): p=1,2, \ldots\right\}+M_{0} .
\end{gathered}
$$

This implies that

$$
\cup\left\{A_{p}\left(F_{i}\right): p=1,2, \ldots\right\} \subset B\left(\theta, M_{0}+\sup \left\{\rho\left(\theta, A_{p}(\theta)\right): p=1,2, \ldots\right\}\right)
$$

and that the set $F_{i+1}$ is bounded. Thus we have shown by induction that all the sets $F_{0}, \ldots, F_{n_{0}}$ are bounded, as asserted. 
Next, choose $s_{0}>s$ such that

$$
F_{i} \subset B\left(\theta, s_{0}\right), i=0, \ldots, n_{0} .
$$

There exists a neighborhood $\mathcal{U}$ of $\left\{A_{t}\right\}_{t=1}^{\infty}$ in $\mathcal{M}$ such that the following property holds:

(ii) for every sequence of mappings $\left\{B_{t}\right\}_{t=1}^{\infty} \in \mathcal{U}$, every point $x \in B\left(\theta, s_{0}\right)$ and every integer $t \geq 1$, we have

$$
\rho\left(B_{t}(x), A_{t}(x)\right) \leq \delta
$$

Assume that

$$
\left\{B_{t}\right\}_{t=1}^{\infty} \in \mathcal{U}, r:\{1,2, \ldots\} \rightarrow\{1,2, \ldots\} \text { and } x \in B(\theta, s) .
$$

We claim that for all integers $i=1, \ldots, n_{0}$,

$$
\prod_{j=1}^{i} B_{r(j)}(x) \in F_{i}
$$

Property (ii), (3.1), (3.2) and (3.5) imply that (3.6) is indeed true for $i=1$.

Assume now that $i<n_{0}$ is a nonnegative integer and that (3.6) holds. Then it follows from property (ii), (3.2), (3.4), (3.5) and (3.6) that

$$
\begin{gathered}
\rho\left(B_{r(i+1)} \prod_{j=1}^{i} B_{r(j)}(x), A_{r(i+1)} \prod_{j=1}^{i} B_{r(j)}(x)\right) \leq \delta \\
\rho\left(B_{r(i+1)} \prod_{j=1}^{i} B_{r(j)}(x), A_{r(i+1)}\left(F_{i}\right)\right) \leq \delta \leq 1
\end{gathered}
$$

and that

$$
\prod_{j=1}^{i+1} B_{r(j)}(x) \in F_{i+1}
$$

Thus we have shown by induction that for all integers $i=1, \ldots, n_{0}$, inclusion (3.6) indeed holds. When combined with (3.4), this implies that

$$
\prod_{j=1}^{i} B_{r(j)}(x) \in B\left(\theta, s_{0}\right), i=1, \ldots, n_{0} .
$$

It follows from property (ii) and inclusion (3.8) that for all integers $i=0, \ldots, n_{0}-1$ and every point $y \in B(\theta, s)$, we have

$$
\rho\left(B_{r(i+1)} \prod_{j=1}^{i} B_{r(j)}(y), A_{r(i+1)} \prod_{j=1}^{i} B_{r(j)}(y)\right) \leq \delta
$$


(here we assume that $\prod_{j=1}^{i} B_{r(j)}=I$ if $i=0$ ). Thus we have shown that if (3.5) holds and $y \in B(\theta, s)$, then the sequences $\left\{x_{i}\right\}_{i=0}^{n_{0}}$ and $\left\{y_{i}\right\}_{i=0}^{n_{0}}$ defined by

$$
x_{0}=x, y_{0}=y, x_{i}=\prod_{j=1}^{i} B_{r(j)}(x), y_{i}=\prod_{j=1}^{i} B_{r(j)}(y), i=1, \ldots, n_{0},
$$

satisfy the conditions assumed in property (i). This leads to the inequality

$$
\epsilon \geq \rho\left(x_{n_{0}}, y_{n_{0}}\right)=\rho\left(\prod_{j=1}^{n_{0}} B_{r(j)}(x), \prod_{j=1}^{n_{0}} B_{r(j)}(y)\right),
$$

which in its turn implies that

$$
\epsilon \geq \rho\left(\prod_{j=1}^{n} B_{r(j)}(x), \prod_{j=1}^{n} B_{r(j)}(y)\right)
$$

for each integer $n \geq n_{0}$. This completes the proof of Theorem 3.1.

\section{Generic result}

Theorem 4.1. There exists a set $\mathcal{F} \subset \mathcal{M}_{n}$, which is a countable intersection of open and everywhere dense subsets of $\mathcal{M}_{n}$, such that every sequence of mappings $\left\{A_{t}\right\}_{t=1}^{\infty} \in \mathcal{F}$ possesses the RWEP.

Proof. Let $\left\{A_{t}\right\}_{t=1}^{\infty} \in \mathcal{M}_{n}$ and $\gamma \in(0,1)$. For every natural number $t$, define

$$
A_{t}^{(\gamma)}(x):=(1-\gamma) A_{t}(x) \oplus \gamma \theta, x \in K .
$$

In view of (1.1) and (4.1), for every natural number $t$ and every pair of points $x, y \in K$, we have

$$
\begin{gathered}
\rho\left(A_{t}^{(\gamma)}(x), A_{t}^{(\gamma)}(y)\right) \\
=\rho\left((1-\gamma) A_{t}(x) \oplus \gamma \theta,(1-\gamma) A_{t}(y) \oplus \gamma \theta\right) \\
\leq(1-\gamma) \rho\left(A_{t}(x), A_{t}(y)\right) \leq(1-\gamma) \rho(x, y) .
\end{gathered}
$$

This implies that $\left\{A_{t}^{(\gamma)}\right\}_{t=1}^{\infty} \in \mathcal{M}$. By (4.1), for every natural number $t \geq 1$, we have

$$
\rho\left(A_{t}^{(\gamma)}(\theta), \theta\right)=\rho\left((1-\gamma) A_{t}(\theta) \oplus \gamma \theta, \theta\right) \leq(1-\gamma) \rho\left(A_{t}(\theta), \theta\right) .
$$

This implies that $\left\{A_{t}^{(\gamma)}\right\}_{t=1}^{\infty} \in \mathcal{M}_{n}$.

We claim that $\left\{A_{t}^{(\gamma)}\right\}_{t=1}^{\infty}$ has the RWEP. To this end, let $\epsilon, s$ be positive numbers. Choose an integer $n_{0} \geq 1$ for which

$$
2 s(1-\gamma)^{n_{0}}<\epsilon
$$


Assume that $r:\left\{1, \ldots, n_{0}\right\} \rightarrow\{1,2, \ldots\}$ and that $x, y \in B(\theta, s)$. It follows from (4.2) and (4.3) that

$$
\begin{gathered}
\rho\left(A_{r\left(n_{0}\right)}^{(\gamma)} \cdots A_{r(1)}^{(\gamma)}(x), A_{r\left(n_{0}\right)}^{(\gamma)} \cdots A_{r(1)}^{(\gamma)}(y)\right) \\
\leq(1-\gamma)^{n_{0}} \rho(x, y) \leq 2 s(1-\gamma)^{n_{0}}<\epsilon .
\end{gathered}
$$

Therefore $\left\{A_{t}^{(\gamma)}\right\}_{t=1}^{\infty}$ indeed possesses the RWEP, as claimed.

By (4.1), for every natural number $t$ and every point $x \in K$, we have

$$
\rho\left(A_{t}^{(\gamma)}(x), A_{t}(x)\right)=\rho\left((1-\gamma) A_{t}(x) \oplus \gamma \theta, A_{t}(x)\right) \leq \gamma \rho\left(A_{t}(x), \theta\right) .
$$

We claim that

$$
\left\{A_{t}^{(\gamma)}\right\}_{t=1}^{\infty} \rightarrow\left\{A_{t}\right\}_{t=1}^{\infty} \text { in } \mathcal{M}_{n} \text { as } \gamma \rightarrow 0^{+}
$$

Let $\epsilon$ be a positive number and let $m \geq 1$ be an integer. Choose a number $\gamma_{0} \in(0,1)$ satisfying

$$
\gamma_{0}\left(m+\sup \left\{\rho\left(A_{t}(\theta), \theta\right): t=1,2, \ldots\right\}\right)<\epsilon .
$$

Assume that $\gamma \in\left(0, \gamma_{0}\right)$. In view of (4.1), (4.4) and (4.5), for every point $x \in B(\theta, m)$ and each natural number $t$, we have

$$
\begin{gathered}
\rho\left(A_{t}^{(\gamma)}(x), A_{t}(x)\right) \leq \gamma \rho\left(A_{t}(x), \theta\right) \\
\leq \gamma_{0}\left(\rho\left(A_{t}(x), A_{t}(\theta)\right)+\rho\left(A_{t}(\theta), \theta\right)\right) \\
\leq \gamma_{0}\left(\left(\rho(x, \theta)+\rho\left(A_{t}(\theta), \theta\right)\right) \leq \gamma_{0}\left(m+\rho\left(A_{t}(\theta), \theta\right)\right)<\epsilon .\right.
\end{gathered}
$$

Thus the set

$$
\left\{\left\{A_{t}^{(\gamma)}\right\}_{t=1}^{\infty}:\left\{A_{t}\right\}_{t=1}^{\infty} \in \mathcal{M}_{n}, \gamma \in(0,1)\right\}
$$

is everywhere dense in $\mathcal{M}_{n}$ and its elements possess the RWEP.

Let $\left\{A_{t}\right\}_{t=1}^{\infty} \in \mathcal{M}_{n}, \gamma \in(0,1)$ and let $q \geq 1$ be an integer. Theorem 3.1 implies that there exist an integer $n\left(\left\{A_{t}\right\}_{t=1}^{\infty}, \gamma, q\right) \geq 1$ and an open neighborhood $\mathcal{U}\left(\left\{A_{t}\right\}_{t=1}^{\infty}, \gamma, q\right)$ of $\left\{A_{t}^{(\gamma)}\right\}_{t=1}^{\infty}$ in the metric space $\mathcal{M}_{n}$ such that the following property holds:

(i) for every sequence of mappings $\left\{B_{t}\right\}_{t=1}^{\infty} \in \mathcal{U}\left(\left\{A_{t}\right\}_{t=1}^{\infty}, \gamma, q\right)$, every mapping $r:\{1,2, \ldots\} \rightarrow\{1,2, \ldots\}$, every pair of points $x, y \in B(\theta, q)$ and every natural number $n \geq n\left(\left\{A_{t}\right\}_{t=1}^{\infty}, \gamma, q\right)$, we have

$$
\rho\left(B_{r(n)} \cdots B_{r(1)}(x), B_{r(n)} \cdots B_{r(1)}(y)\right) \leq q^{-1} .
$$

Now define

$$
\begin{aligned}
\mathcal{F}:= & \cap_{p=1}^{\infty} \cup\left\{\mathcal{U}\left(\left\{A_{t}\right\}_{t=1}^{\infty}, \gamma, q\right):\left\{A_{t}\right\}_{t=1}^{\infty} \in \mathcal{M}_{n}\right. \\
& \gamma \in(0,1), q \geq p \text { is an integer }\}
\end{aligned}
$$

It is clear that $\mathcal{F}$ is a countable intersection of open and everywhere dense subsets of $\mathcal{M}_{n}$.

Let

$$
\left\{B_{t}\right\}_{t=1}^{\infty} \in \mathcal{F}, s>0 \text { and } \epsilon>0
$$


Choose a natural number $p$ such that

$$
p>s+\epsilon^{-1} .
$$

In view of (4.6) and (4.7), there exist

$$
\left\{A_{t}\right\}_{t=1}^{\infty} \in \mathcal{M}_{n}, \gamma \in(0,1), \text { and } q \in\{p, p+1, \ldots\}
$$

such that

$$
\left\{B_{t}\right\}_{t=1}^{\infty} \in \mathcal{U}\left(\left\{A_{t}\right\}_{t=1}^{\infty}, \gamma, q\right) .
$$

It follows from property (i), (4.8) and (4.9) that for every mapping

$$
r:\{1,2, \ldots,\} \rightarrow\{1,2, \ldots\},
$$

every pair of points

$$
x, y \in B(\theta, s) \subset B(\theta, q)
$$

and every integer

$$
\begin{gathered}
n \geq n\left(\left\{A_{t}\right\}_{t=1}^{\infty}, \gamma, q\right), \\
\rho\left(\prod_{i=1}^{n} B_{r(i)}(x), \prod_{i=1}^{n} B_{r(i)}(y)\right) \leq q^{-1} \leq p^{-1}<\epsilon .
\end{gathered}
$$

Thus $\left\{B_{t}\right\}_{t=1}^{\infty}$ has the RWEP. This completes the proof of Theorem 4.1.

\section{Acknowledgments.}

The first author was partially supported by the Israel Science Foundation (Grant No. 389/12), by the Fund for the Promotion of Research at the Technion and by the Technion General Research Fund. Both authors are grateful to an anonymous referee for many helpful suggestions.

\section{References}

[1] H.H. Bauschke, J.M. Borwein, On projection algorithms for solving convex feasibility problems, SIAM Review 38 (1996) 367-426.

[2] H.H. Bauschke, J.M. Borwein and A.S. Lewis, The method of cyclic projections for closed convex sets in Hilbert space, Recent Developments in Optimization Theory and Nonlinear Analysis, Contemporary Mathematics 204 (1997) 1-38.

[3] Y. Censor, S. Reich, Iterations of paracontractions and firmly nonexpansive operators with applications to feasibility and optimization, Optimization 37 (1996) 323-339.

[4] J.E. Cohen, Ergodic theorems in demography, Bull. Amer. Math. Soc. 1 (1979) 275-295. 
[5] J. Dye, S. Reich, Random products of nonexpansive mappings, Optimization and Nonlinear Analysis, Pitman Research Notes in Mathematics Series 244 (1992) 106-118.

[6] K. Goebel, S. Reich, Uniform Convexity, Hyperbolic Geometry, and Nonexpansive Mappings, Marcel Dekker, New York and Basel 1984.

[7] S. Reich, The alternating algorithm of von Neumann in the Hilbert ball, Dynamic Systems Appl. 2 (1993) 21-25.

[8] S. Reich, I. Shafrir, Nonexpansive iterations in hyperbolic spaces, Nonlinear Analysis 15 (1990) 537-558.

[9] S. Reich, A.J. Zaslavski, Convergence of generic infinite products of nonexpansive and uniformly continuous operators, Nonlinear Analysis 36 (1999) 1049-1065.

[10] S. Reich, A.J. Zaslavski, Generic aspects of metric fixed point theory, Handbook of Metric Fixed Point Theory, Kluwer, Dordrecht 2001 557-575.

[11] S. Reich, A.J. Zaslavski, Genericity in Nonlinear Analysis, Developments in Mathematics 34, Springer, New York 2014.

[12] S. Reich, A.J. Zaslavski, Asymptotic behavior of infinite products of nonexpansive mappings, J. Nonlinear Convex. Anal. 17 (2016) 1967-1973.

[13] S. Reich and A.J. Zaslavski, Asymptotic behavior of generic infinite products of nonexpansive mappings, J. Nonlinear Convex Anal. 18 (2017) 17-27.

\title{
DOI: $10.7862 /$ rf.2017.10
}

\section{Simeon Reich}

email: sreich@tx.technion.ac.il

Department of Mathematics

The Technion - Israel Institute of Technology

32000 Haifa

ISRAEL

\author{
Alexander J. Zaslavski \\ email: ajzasl@tx.technion.ac.il \\ Department of Mathematics \\ The Technion - Israel Institute of Technology \\ 32000 Haifa \\ ISRAEL
}

Received 23.10.2016

Accepted 12.02.2017 